\title{
Performance and early science with the Subaru Coronagraphic Extreme Adaptive Optics project
}

Thayne Currie, Olivier Guyon, Julien Lozi, Tyler Groff, N. Jeremy Kasdin, et al.

Thayne Currie, Olivier Guyon, Julien Lozi, Tyler Groff, N. Jeremy Kasdin, Frantz Martinache, Timothy D. Brandt, Jeffrey Chilcote, Christian Marois, Benjamin Gerard, Nemanja Jovanovic, Sébastien Vievard, "Performance and early science with the Subaru Coronagraphic Extreme Adaptive Optics project," Proc. SPIE 11117, Techniques and Instrumentation for Detection of Exoplanets IX, 111170X (9 September 2019); doi: 10.1117/12.2529689

SPIE Event: SPIE Optical Engineering + Applications, 2019, San Diego, California, United States 


\title{
Performance and early science with the Subaru Coronagraphic Extreme Adaptive Optics project
}

\author{
Thayne Currie ${ }^{\mathrm{a}, \mathrm{b}}$, Olivier Guyon ${ }^{\mathrm{b}}$, Julien Lozi ${ }^{\mathrm{b}}$, Tyler Groff ${ }^{\mathrm{c}}$, N. Jeremy Kasdin ${ }^{\mathrm{d}}$, Frantz \\ Martinache $^{\mathrm{e}}$, Timothy D Brandt ${ }^{\mathrm{f}}$, Jeffrey Chilcote ${ }^{\mathrm{g}}$, Christian Marois ${ }^{\mathrm{h}, \mathrm{i}}$, Benjamin Gerard ${ }^{\mathrm{i}}$, \\ Nemanja Jovanovic ${ }^{j}$, and Sebastien Vievard ${ }^{b}$ \\ ${ }^{a}$ NASA-Ames Research Center, Moffett Field, California, USA \\ bSubaru Telescope, 650 N. Aohoku Pl., Hilo, Hawai'i, USA \\ cNASA-Goddard Space Flight Center, Greenbelt, MD, USA \\ dPrinceton University, Princeton, NJ, USA

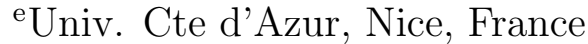 \\ ${ }^{\mathrm{f}}$ University of California-Santa Barbara, Santa Barbara, CA, USA \\ gUniversity of Notre Dame, South Bend, IN, USA \\ ${ }^{\mathrm{h}} \mathrm{NRC}-$ Herzberg, Victoria, BC, Canada \\ ${ }^{i}$ University of Victoria, BC, Canada \\ ${ }^{\mathrm{j} C a l i f o r n i a}$ Institute of Technology, Pasadena, CA, USA
}

\begin{abstract}
We describe the current performance of the Subaru Coronagraphic Extreme Adaptive Optics (SCExAO) instrument on the Subaru telescope on Maunakea, Hawaii and present early science results for SCExAO coupled with the CHARIS integral field spectrograph. SCExAO now delivers H band Strehl ratios up to $\sim 0.9$ or better, extreme AO corrections for optically faint stars, and planet-to-star contrasts rivaling that of GPI and SPHERE. CHARIS yield high signal-to-noise detections and 1.1-2.4 $\mu \mathrm{m}$ spectra of benchmark directly-imaged companions like HR 8799 cde and kappa And b that clarify their atmospheric properties. We also show how recently published as well as unpublished observations of LkCa 15 lead to a re-evaluation of its claimed protoplanets. Finally, we briefly describe plans for a SCExAO-focused direct imaging campaign to directly image and characterize young exoplanets, planet-forming disks, and (later) mature planets in reflected light.
\end{abstract}

Keywords: adaptive optics, extrasolar planets, infrared

\section{INTRODUCTION}

Starting about a decade ago, ground-based telescopes using facility (conventional) adaptive optics (AO) systems revealed the first direct images of young, self-luminous superjovian mass planets orbiting nearby stars. ${ }^{1-5}$ Followup multi-wavelength photometry provided the first constraints on their atmospheric properties, revealing the planets to be redder and cloudier/dustier than substellar objects with the same temperatures and, in some cases, showing evidence for non-equilibrium carbon chemistry. ${ }^{6,7}$ Spectra for the first directly-imaged planets revealed evidence for low surface gravities and the presence of multiple molecular species. ${ }^{5,8}$ Near-infrared (IR) conventional AO systems achieve typical planet-to-star contrasts typically of $10^{-3}, 10^{-4}$, and $10^{-5}$ to $5 \times 10^{-6}$ at angular separations of $0.25 ", 0.5 "$ and 1.0". ${ }^{9}$ Most imaged exoplanets discovered with conventional AO lie beyond 0.5 ". Surveys with these systems are typically sensitive to only the most massive planets $\left(10-15 M_{\mathrm{J}}\right)$ at orbits exterior to those in our own solar system $\left(a_{\mathrm{p}} \gtrsim 30-100 \mathrm{au}\right) .{ }^{10}$

Now, extreme AO systems like the Gemini Planet Imager (GPI) on Gemini-South and Spectro-Polarimetric High-contrast Exoplanet REsearch instrument (SPHERE) on the Very Large Telescope (VLT) are able to detect planets at $0.25 "-1.0 "$ a factor of 10-100 times fainter in the near-IR. ${ }^{11,12}$ These deeper contrasts have opened

Further author information: (Send correspondence to T. Currie)

T. Currie: E-mail: currie@naoj.org

Techniques and Instrumentation for Detection of Exoplanets IX, edited by Stuart B. Shaklan, Proc. of SPIE Vol. 11117, 111170X - (C) 2019 SPIE · CCC code: 0277-786X/19/\$21 - doi: 10.1117/12.2529689 
up new exoplanet discovery space, probing planets that are lower mass (down to $\sim 2 M_{\mathrm{J}}$ ) and closer in angular separation $(\rho \sim 0.1 "-0.4 ")$ and physical projected separation $\left(a_{\mathrm{p}} \sim 10-15 \mathrm{au}\right) .{ }^{13-17}$ Integral field spectrographs coupled to GPI and SPHERE have provided new insights into the atmospheric properties of young, jovian planets, including their cloud cover, temperature, and gravities (e.g. ${ }^{18-20}$ ). Extreme AO surveys have provided new constraints on the frequency of 5-13 $M_{\mathrm{J}}$ planets at 10-30 au, suggesting that these companions form a separate population from more massive brown dwarf companions. ${ }^{21}$

Despite recent technological advances utilized by these extreme AO systems, only about 20 or so exoplanets have been directly imaged thus far. Direct imaging preferentially detects luminous (massive), wide-separation planets. However, analyses of radial-velocity searches for mature planets suggest that the frequency of planets at the innermost separations probed by the latest extreme AO surveys is small compared to a peak at $\sim 2-3$ au. ${ }^{22}$ Even at wider separations (10-30 au), typical sensitivities are on the order of $\sim 3-5 M_{\mathrm{J}} .{ }^{21}$ Yet the frequency of gas giant planets comparable to or lower than Jupiter's mass is significantly higher than that of superjovian planets. ${ }^{22}$ The yield of direct imaging surveys appears to be biased towards stars more massive than the Sun. Directly detecting planets on smaller orbits, with lower masses, and around a wider range of stellar masses requires significantly better performance at small angular separations, a higher-fidelity AO correction at wider separations, and better performance for optically fainter stars.

Here, we provide an update on the performance and early science obtained with the Subaru Coronagraphic Extreme Adaptive Optics project (SCExAO) at the Subaru Telescope on Maunakea. ${ }^{23}$ SCExAO employs improvements in wavefront control hardware and software improving its performance at small angular separations and for faint guide stars. It will mature key advances in wavefront control and coronagraphy needed to move beyond recent exoplanet direct imaging capabilities to those capable of imaging an Earth with ELTs.

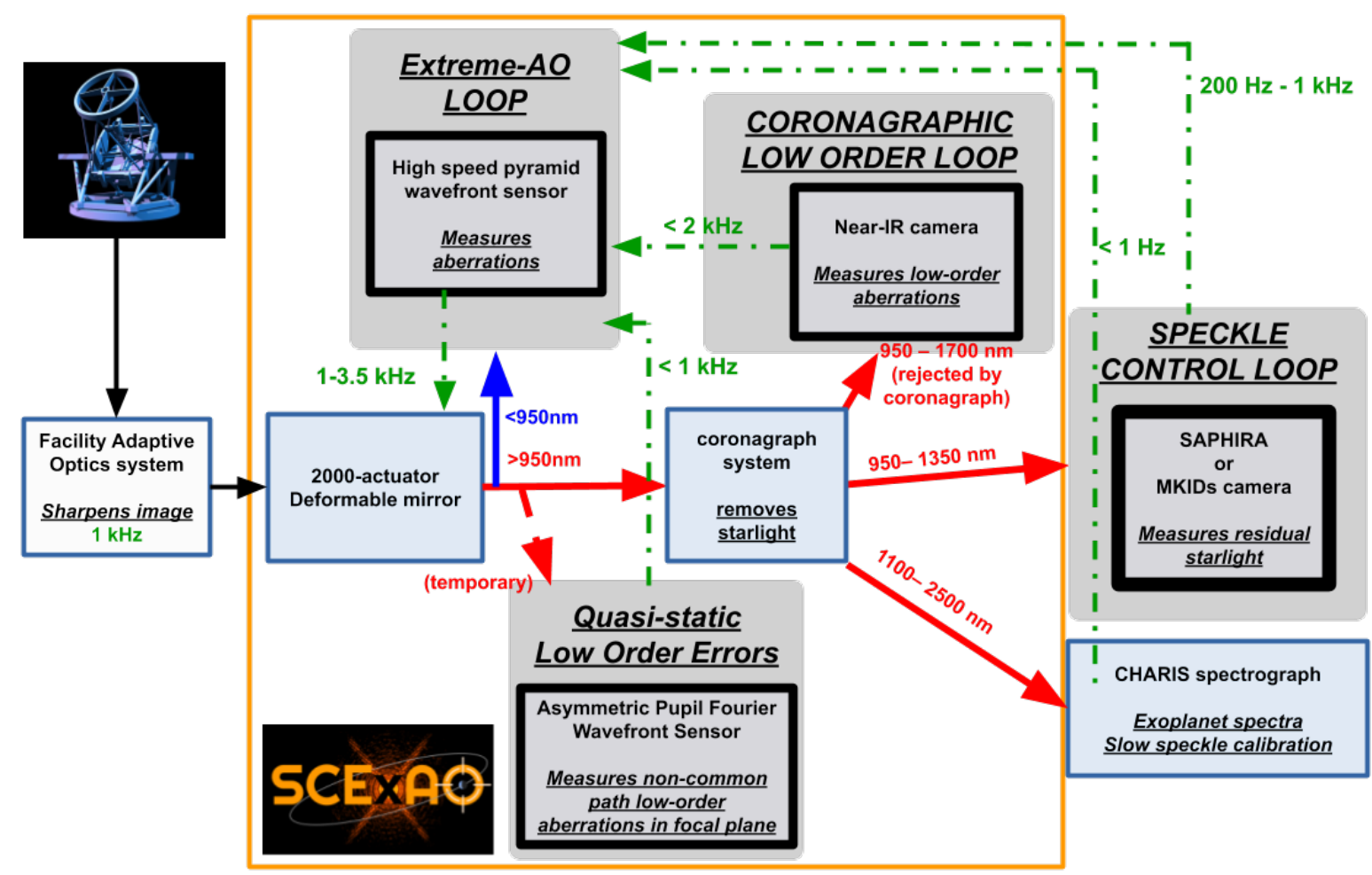

Figure 1. Current schematic of SCExAO. Note that the coronagraphic low-order loop is not in normal operation; the MKIDs camera (MEC) is undergoing commissioning. 


\section{SCEXAO DESIGN AND PERFORMANCE}

The standard mode of SCExAO science operation is shown in Figure 1. The system takes in and further sharpens partially-corrected light from Subaru's facility AO system (A0-188) as input, which typically achieves $~ 30-40 \%$ Strehl at $1.6 \mu \mathrm{m}$. SCExAOs main wavefront control loop includes a 2,000-element MEMS deformable mirror from Boston MicroMachines driven by a modulated Pyramid wavefront sensor using an $\mathrm{OCAM}^{2} \mathrm{~K}$ camera from First-Light Imaging operating over a 600-900 nm bandpass. The loop can run at speeds up to $3.5 \mathrm{kHz}$. However, currently in normal science operations it operates at $2 \mathrm{kHz}$ for bright stars coupled with predictive wavefront control (see below) to decrease wavefront sensor noise error. For very faint $\operatorname{stars}(I>9)$, we typically run the loop at $1 \mathrm{kHz}$. The loop can correct for up to 1200 modes of dynamic aberrations.

Figure 2 presents the AO performance of SCExAO as a function of atmospheric conditions and $I$-band magnitude. For extremely bright stars $(I=1-5)$, SCExAO can deliver excellent AO corrections, with estimated Strehl ratios (at $1.6 \mu \mathrm{m}$ ) of 0.9-0.94 for slightly above average to top-quartile seeing conditions on Maunakea (left panel). Estimated Strehl ratios drop to $\sim 0.75-0.8$ for much fainter stars $(I \sim 7-8)$, or below for below-average conditions, although in the former case some of the estimated drop may be due to noise in the estimate of the wavefront correction residuals. Under the best conditions $(\theta \sim 0.2$ "-0.5"), SCExAO can deliver sharp AO corrections for stars as faint as $\mathrm{R} \sim 11.4-12$, such as LkCa 15 or V819 Tau (right panel).

Corrected starlight is fed into different optical/near-infrared (IR) science instruments. For most exoplanet imaging programs, the near-IR integral field spectrograph CHARIS is used. CHARIS operates in two modes. The primary workhorse configuration is its low resolution $(\mathrm{R} \sim 20)$ broadband mode covering the three major near-IR bandpasses (J, H, and $\mathrm{K}$ ) simultaneously, which makes the instrument well-suited for exoplanet discovery and coarse spectral characterization. A higher-resolution $(\mathrm{R} \sim 70)$ mode in $\mathrm{J}, \mathrm{H}$, or $\mathrm{K}$ allows for more detailed, followup characterization. For broadband mode, we typically use a Lyot coronagraph with a $\sim 0.1$ " occulting spot; for $H$ band the vector vortex coronagraph is used. Satellite spots placed at 15.5 $\lambda / \mathrm{D}$ are used for spectrophotometric calibration and image registration.

The CHARIS Data Reduction Pipeline converts raw data into data cubes. ${ }^{24}$ There is currently no standardized, public pipeline for subsequent data reduction steps (e.g. image registration, PSF subtraction). However, T.C. has developed data reduction tools for these steps that will likely separately become part of a supported pipeline component. ${ }^{25,26}$ Other collaborators have successfully carried out at least many data reduction steps using the public PyKLIP package ${ }^{27}$ or their own proprietary codes. ${ }^{28,29}$

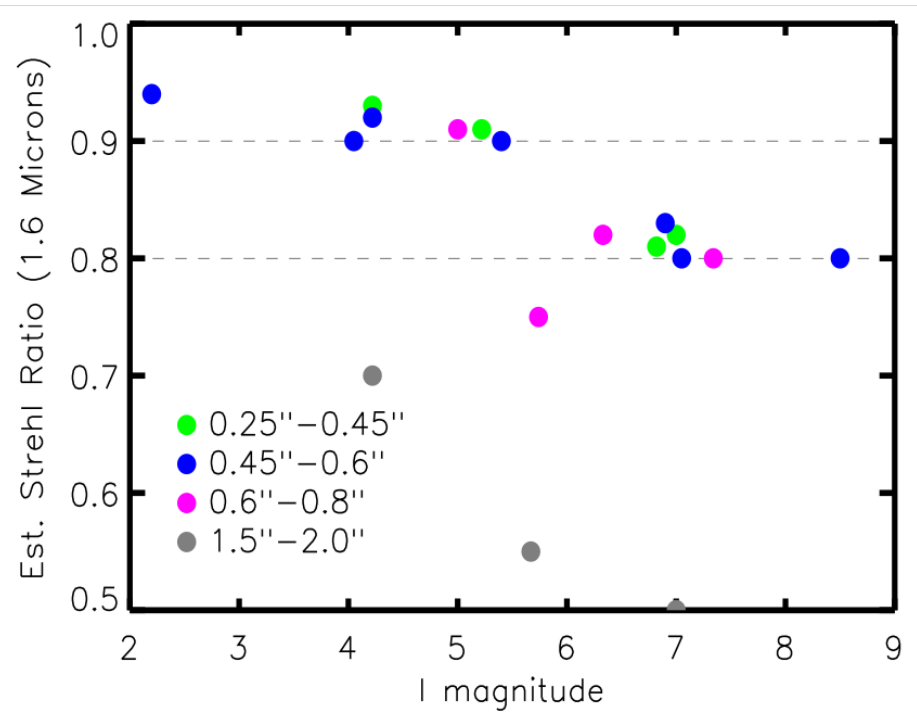

\section{LkCa 15} $R \sim 11.4$

Figure 2. (left) Plot of Strehl ratio - as estimated from the residual wavefront error - vs. guide star $I$ band magnitude. (right) SCExAO PSF for LkCa 15 at $K$ band, an optically faint star revealing numerous Airy rings. 
As shown in 3, the raw contrast achieved with SCExAO/CHARIS under very good correction (S.R. $\sim 0.9)$ is roughly flat between $\sim 0.3$ " and $0.6 "$ at $\approx 10^{-4}, \sim 3 \times 10^{-4}$ at $0.25 "$, and $\sim 2 \times 10^{-5}$ at $0.8 "$. At small separations, SPHERE achieves deeper raw contrast, ${ }^{30}$ plausible due to its more optimal choice of coronagraphs to suppress halo light compared to our use of a standard Lyot coronagraph, among other possible factors.

After using advanced post-processing, it achieves contrasts of $\sim 10^{-5}$ at 0.25 " and $10^{-6}$ at 0.5 " over 1 hour-long sequences and under good conditions. These values are comparable to typical contrasts achieved with the Gemini Planet Imager in $H$ band. Improvements in wavefront control and coronagraphy will likely lead to substantial gains in contrast at small separations.

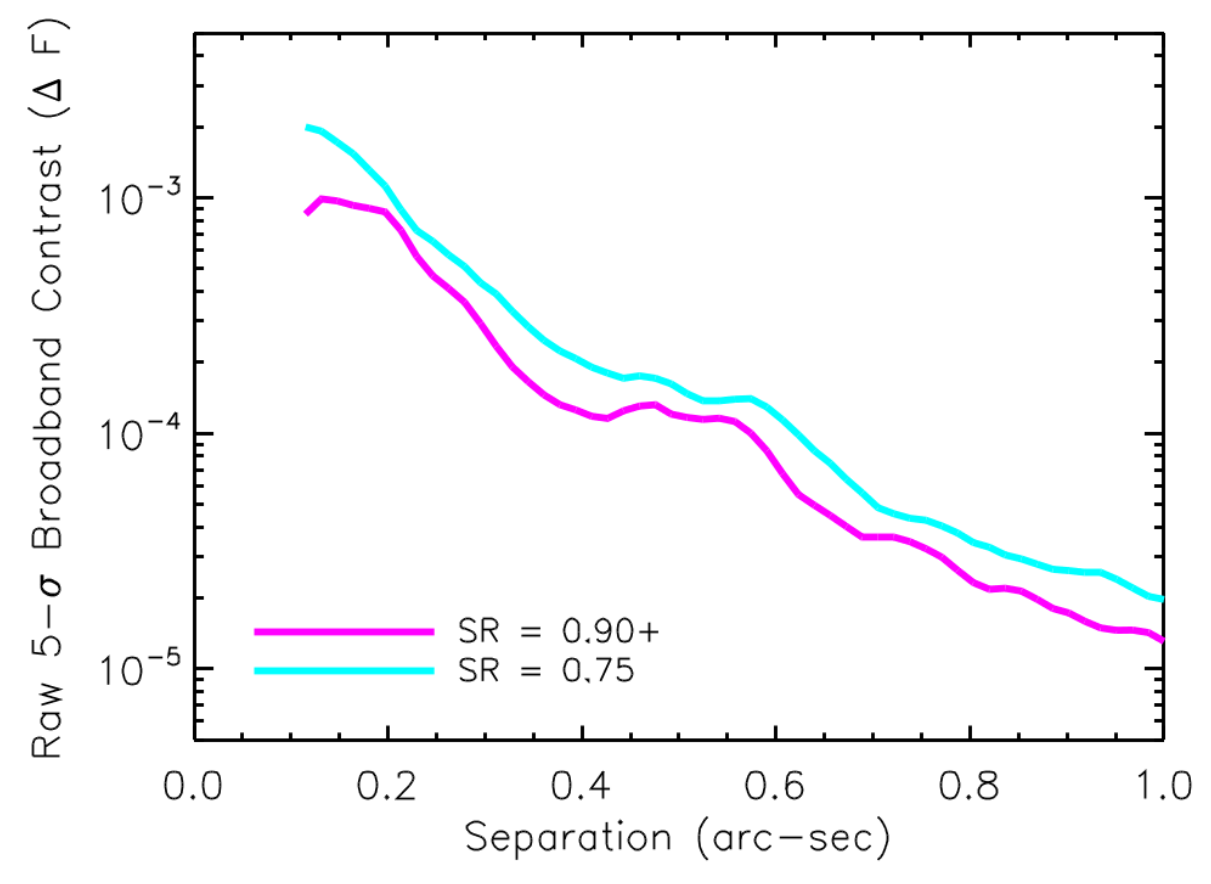

Figure 3. Raw 5- $\sigma$ broadband contrast obtained for stars at S.R. $\sim 0.75$ and $\sim 0.9$ (corrected for finite sample sizes) but without fully masking residual light from satellite spots. Towards the highest Strehl ratios, the raw contrast curve is nearly flat from 0.3 " -0.6 ". The curves are computed by measuring, at each radius, the surface brightness standard deviation (assumed here to be the noise), and multiplying it by 5 .

Figure 4 uses observations of HR 8799 to illustrate the practical performance gain for SCExAO/CHARIS over conventional AO facilties. SCExAO/CHARIS yields a $\sim 23-\sigma$ detection of HR 8799 e - invisible in the Keck/NIRC2 data - and much stronger detections of the outer two planets HR $8799 \mathrm{~cd}$. While spectral differential imaging does result in a contrast gain, a key advantage of SCExAO/CHARIS appears to be the relatively strong temporal correlation of the halo, making post-processing with angular differential imaging (ADI; ${ }^{31}$ ) more effective. ${ }^{29}$

\section{EARLY SCEXAO/CHARIS SCIENCE RESULTS}

The first year of full science operations for SCExAO and CHARIS has yielded seven peer-reviewed publications ${ }^{25,26,29,33-36}$ providing new insights about massive exoplanets, planet candidates, protoplanetary and debris disks, brown dwarfs and low-mass stars, and the efficacy of advanced coronagraphy and PSF subtraction methods. Here, we focus on two systems $-\kappa$ Andromedae and LkCa $15-$ for which SCExAO/CHARIS data provided new key insights about (proposed) planetary companions. 


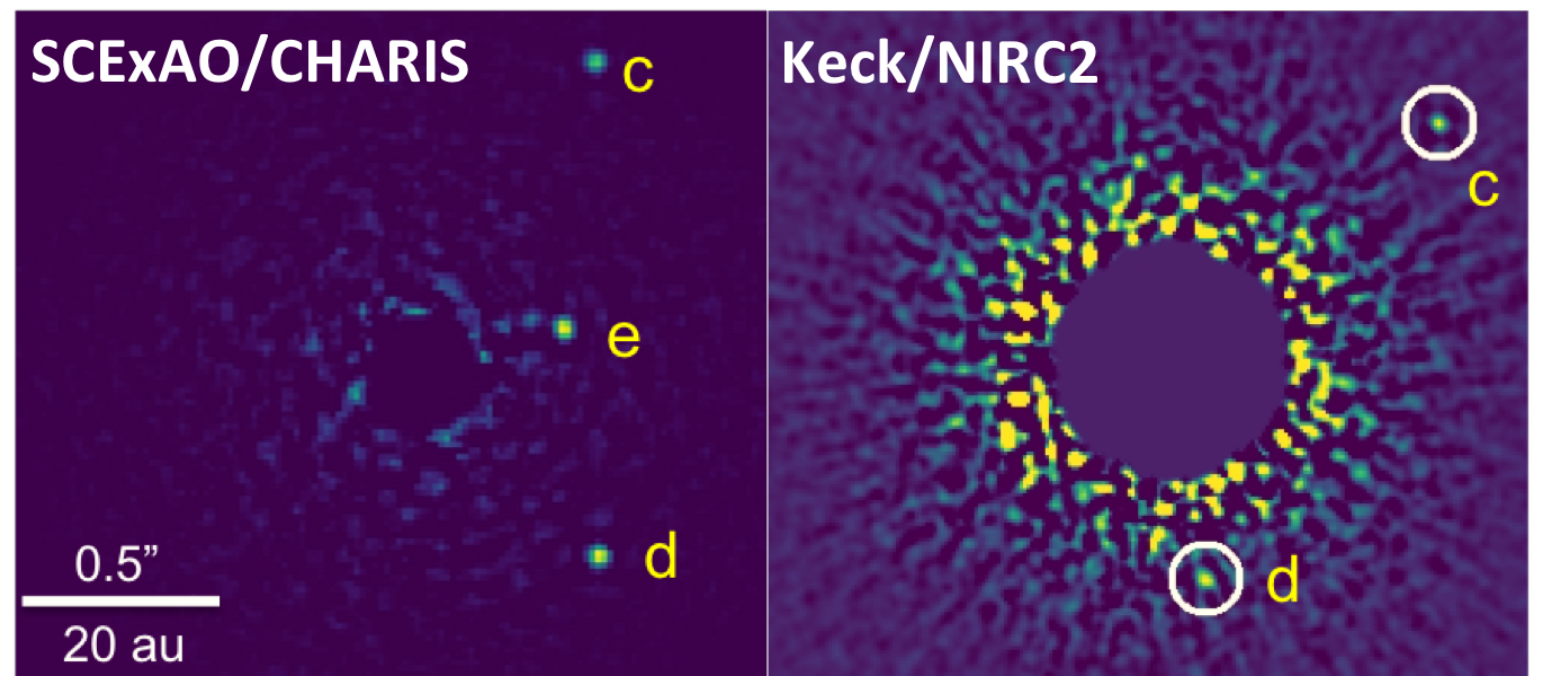

Figure 4. (left) SCExAO/CHARIS HR 8799 image (wavelength-collapsed) compared to Keck/NIRC2 $H$ band image (right), showing stronger detections of HR $8799 \mathrm{~cd}$ and detection of HR $8799 \mathrm{e}$. HR 8799 b lies outside CHARIS's field of view. The CHARIS data were reduced first using advanced PSF subtraction ${ }^{32}$ to remove the halo in angular differential imaging (ADI) and then simple classical spectral different imaging (a median-combination of channels magnified by wavelength). The Keck/NIRC2 data were reduced in ADI only.

\subsection{SCExAO Observations of $\kappa$ And}

The directly-imaged low-mass companion to the B9V star $\kappa$ Andromedae ( $\kappa$ And b; ${ }^{37}$ ) is an object whose properties were better constrained with SCExAO/CHARIS data. Depending on whether the system $\kappa$ And was a young star with an age (i.e. 30-40 Myr) comparable to moving groups like the Columba association or an older, $\sim 200$ Myr-old one, $\kappa$ And b could have a planet-like mass or one consistent with low-mass brown dwarfs. ${ }^{37,38}$ Interferometric observations of the star favor a young age. ${ }^{39}$ A spectrum could better determine whether $\kappa$ And b bears a greater resemblance to young, planet-mass brown dwarfs or intermediate-aged more massive brown dwarfs.

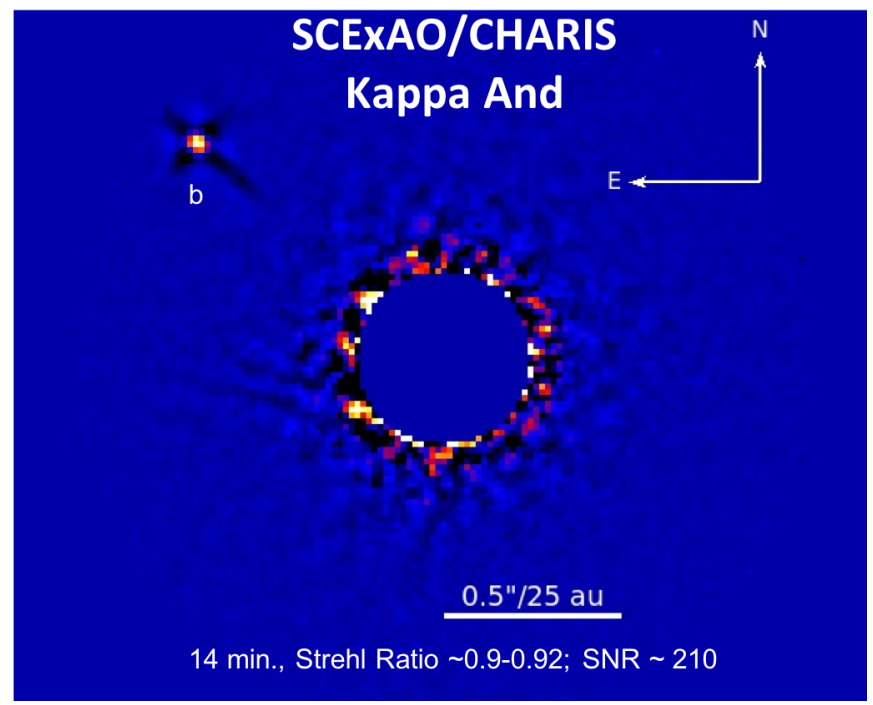

Figure 5. SCExAO/CHARIS image of $\kappa$ And $\mathrm{b}$ from. ${ }^{25}$ 
SCExAO/CHARIS data yielded an exceptionally high SNR JHK spectrum of $\kappa$ And $\mathrm{b}$ and achieved deep contrast limits down to 0.25 " ( $12.5 \mathrm{au})$ despite only 14 minutes of integration time (Figure 5$)$. The shape of $\kappa$ And b's spectrum was best matched by young L0-L1 dwarfs (Figure 6 ). The $H$-band peak of $\kappa$ And b showed strong evidence for the companion being a low gravity object, consistent with a planetary mass.
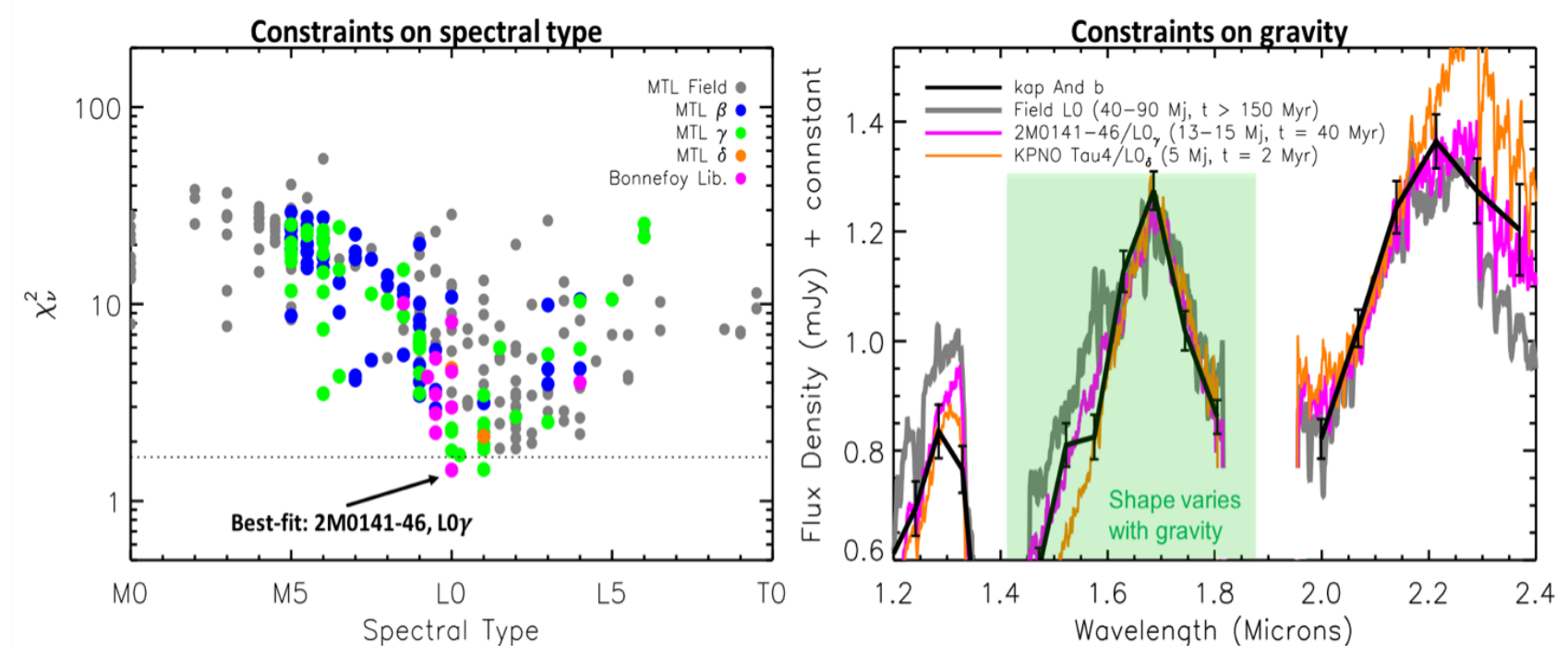

Figure 6. Spectral characterization of $\kappa$ And b with SCExAO/CHARIS, constraining spectral type (left) and gravity (right).

Recent spectral energy distribution modeling of the CHARIS spectrum along with complementary photometry at $Y$ band and in the thermal infrared constrain its temperature to be 1700-1900 $\mathrm{K}$ and favor a surface gravity of $\log (\mathrm{g}) \sim 4.0-4.5$ (Uyama et al. 2019, submitted). Orbital modeling of $\kappa$ And b astrometry suggests that the companion has a high eccentricity and a semimajor axis greater than $\sim 75$ au. As $\kappa$ And b is at a separation where forming massive planets by core accretion is exceptionally difficult, it likely formed through other means, such as gravitational instability. ${ }^{40}$

\subsection{SCExAO Observations of LkCa 15}

The infant Sun-like star LkCa 15 has long been regarded as a key laboratory for studying planet formation. The star's massive, gaseous protoplanetary disk contains multiple dust components, where a sub-au scale hot disk and cold outer disk are responsible for most of the system's broadband infrared emission. ${ }^{41}$ The spatially resolved cavity separating these two dominant components ${ }^{42}$ is a tell-tale sign that much of the disk material has already been incorporated into massive, still-forming "protoplanets.

Two studies using sparse aperture masking interferometry and a separate $H_{\alpha}$ imaging identified up to three protoplanets orbiting $\mathrm{LkCa} 15^{43,44}$ (LkCa 15 'bcd'). However, recent polarimetric imaging reveal additional dust material at separations comparable to LkCa $15 \mathrm{bcd}$, whose scattered light signal could possibly be misinterpreted as planets. SCExAO/CHARIS observations from September 2017 were of sufficient quality to directly detect planets or disk material around LkCa 15, despite the star's faint optical brightness.

SCExAO/CHARIS data and complementary Keck/NIRC2 data spatially resolved the inner regions of LkCa 15 over same wavelengths where LkCa 15 bcd were originally identified. However, they showed that emission attributed LkCa 15 bcd predominantly comes from the arc-like forward-scattering peak of the inner disk component previously seen in polarimetry ${ }^{33}$ (Figure 7 ). The emission has the same brightness previously attributed to planets around LkCa 15 in. ${ }^{44}$

Forward-models of LkCa 15 bcd are inconsistent with the SCExAO/CHARIS and Keck/NIRC2 data. However, scattered light disk models generally reproduced the morphology of emission seen by both instruments. 


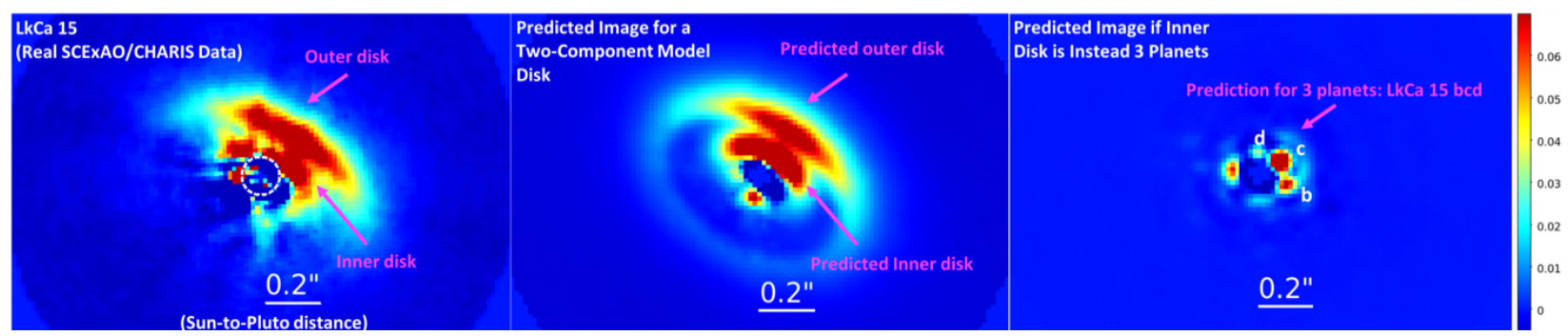

Figure 7. Image of LkCa 15 obtained with SCExAO/CHARIS on September 7, 2017 (left), forward-modeled image for a two-component disk from a theoretical model (middle), and forward-modeled image for three planets derived from previous aperture masking data.

Complementary data taken with the Keck Observatory helped establish that this arc-like structure is static over time and therefore better consistent with a fixed structure like a disk than orbiting planets.

Unpublished data appear consistent with a disk interpretation for most of the emission seen around LkCa 15 at small angles (Figure 8). SCExAO/HiCIAO K-band data obtained in 2016 likewise show a continuous arc of emission. Using archival Keck/NIRC2 data, we have also successfully resolved the inner and outer disk components at $M_{\mathrm{S}}\left(\lambda_{\mathrm{o}} \sim 4.67 \mu \mathrm{m}\right)$. In both data sets, we find no direct evidence for protoplanets around LkCa 15.

Irrespective of our evidence that emission around $\mathrm{LkCa} 15$ is static, claiming evidence for orbiting protoplanets from aperture masking data is challenging. Astrometric error bars reported for LkCa 15 'bcd' are large. It is possible that variable $u--v$ coverage between epochs can induce apparent astrometric offsets when a binary model is assumed in the image reconstruction process (C. Cacares 2019, in prep.). The choice of calibrators, and whether or not they have circumstellar material, is also important. As our previous study notes, previous aperture masking data for LkCa 15 is not faithful reproducing the spatial structure of emission at small angles. ${ }^{33}$ In other systems with previously claimed protoplanets like HD 169142, structures within the disk could themselves be orbiting. ${ }^{45}$ Distinguishing between planet and disk also requires a forward-model of each emission source through the data.

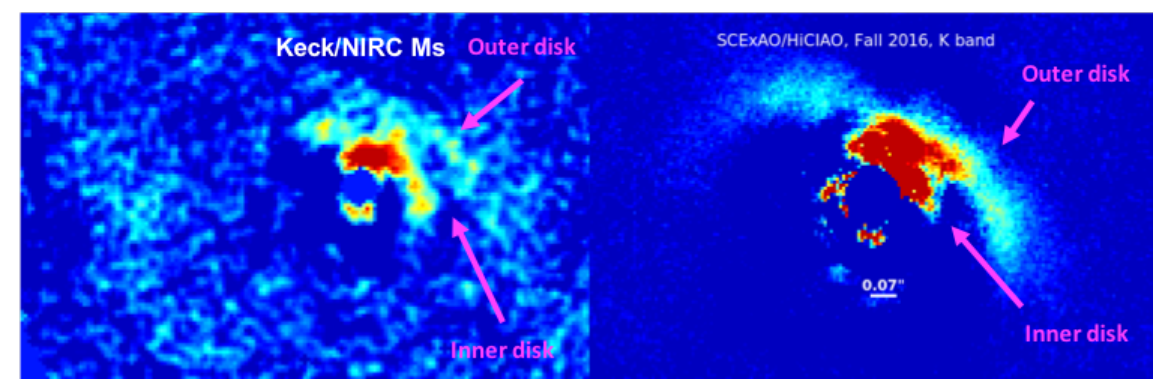

Figure 8. Unpublished SCExAO/HiCIAO and Keck/NIRC2 images of LkCa 15. Both data sets show evidence for two separate disk components but not directly-imaged planets.

Despite the above cautions, it is still possible that future observations will reveal a set of orbiting protoplanets, perhaps even at the locations previously proposed for LkCa 15 'bcd'. Such companions, though, would be significantly fainter than previously reported: e.g. at a contrast of $\sim 10^{-3}$ instead of $\sim 10^{-2}$. Obtaining accurate photometry and astrometry will be challenging given the appearance of such companions against a bright inner disk. 

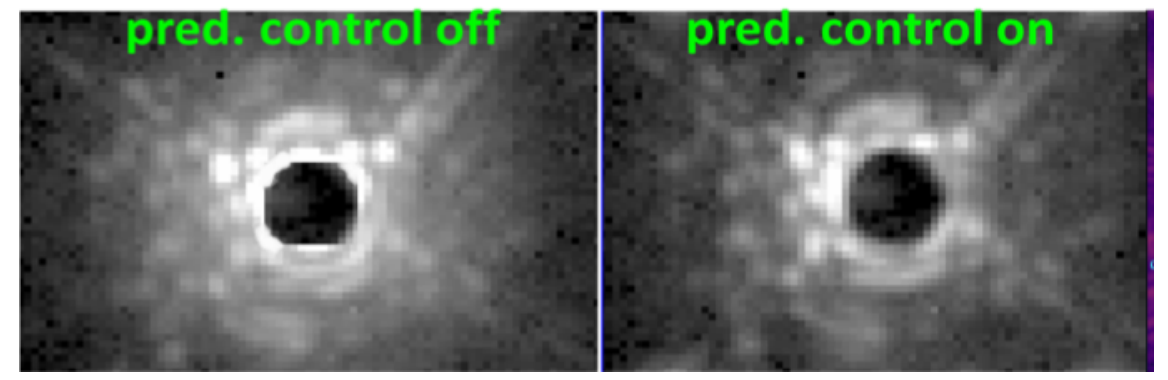

Figure 9. On-sky SCExAO PSF without (left) and with (right) predictive control. The images are of the same star and are averages of 54 consecutive $0.5 \mathrm{~s}$ images taken 3 minutes apart (26s total).

\section{FUTURE PLANS FOR SCEXAO}

\subsection{Technical Advances}

A detailed overview of recent and upcoming technical improvements to SCExAO is discussed in Lozi et al.: ${ }^{46}$ a subset of these upgrades are discussed below, particularly focusing on wavefront sensing/control advances and new science modes.

- Predictive Wavefront Control - We have now successfully implemented "predictive control" using empirical orthogonal functions. ${ }^{47}$ Predictive control allows us to reduce the servo lag error in the wavefront sensing error budget, improving contrast at small angular separations. Figure 9 shows a demonstration of predictive control, where it yields a factor of $\sim 3$ improvement in contrast at small angular separations.

- CRED-2 Detector - We have replaced SCExAO's internal short-wave infrared camera $\left(170 \mathrm{~Hz}, 100 \mathrm{e}^{-}\right.$ read noise) with a new CRED-2 detector from First-Light, which can run in excess of $1 \mathrm{kHz}$ in subframe mode, with low, $<30 \mathrm{e}^{-}$read noise and utilized for focal-plane wavefront control. The CRED-2 allows precise alignment of a coronagraph using both focal plane and pupil plane viewing modes. The CRED-2 can also be used to obtain broadband science images at wavelengths adjacent to those being used by CHARIS.

- MEC - A more fundamental advance is MEC, an 20,000 pixel MKIDS-based detector built by the University of California-Santa Barbara. MEC is a noiseless, ultra-cooled photon-counting detector able to measure the energy and wavelength of every photon. This capable makes MEC a powerful tool for reducing chromatic effects in the speckle halo and for driving fast, focal-plane wavefront sensing and control (e.g. speckle nulling).

- New Focal-Plane Wavefront Sensing Techniques - In addition to speckle nulling, additional focalplane techniques may be tested to allow SCExAO to achieve a far deeper, sustained dark hole than previously possible. In particular, in collaboration with NASA-Ames Research Center, the SCExAO team is developing and eventually planning to implement (on sky) Linear Dark Field Control, which uses the linear response of perturbations in the bright, uncorrected region to maintain a dark hole originally dug through focal-plane wavefront sensing techniques. ${ }^{48}$ See SPIE paper 11117-65 for more details. ${ }^{49}$

- Integral Field Polarimetry with CHARIS - Recently commissioned, CHARIS's "integral field polarimetry" mode splits light into two polarizations over a 1.2"x2.4" field for 22 separate spectral channels covering $\mathrm{J}$ through $\mathrm{K}$. This new mode allows us to detect small-scale emission from protoplanetary and debris disks and yield both total intensity and polarized intensity spectra to get a wavelength-dependent polarization fraction. Its utility for planet detection around young stars is key. Specifically, it can allow us to measure both total and polarized intensity will help us to determine whether candidate point source-like features in disks are highly polarized. Having total intensity IFS data for any point sources detected will also be critical to help characterize these sources, to assess whether their spectra look like substellar objects or CPD-dominated objects. 


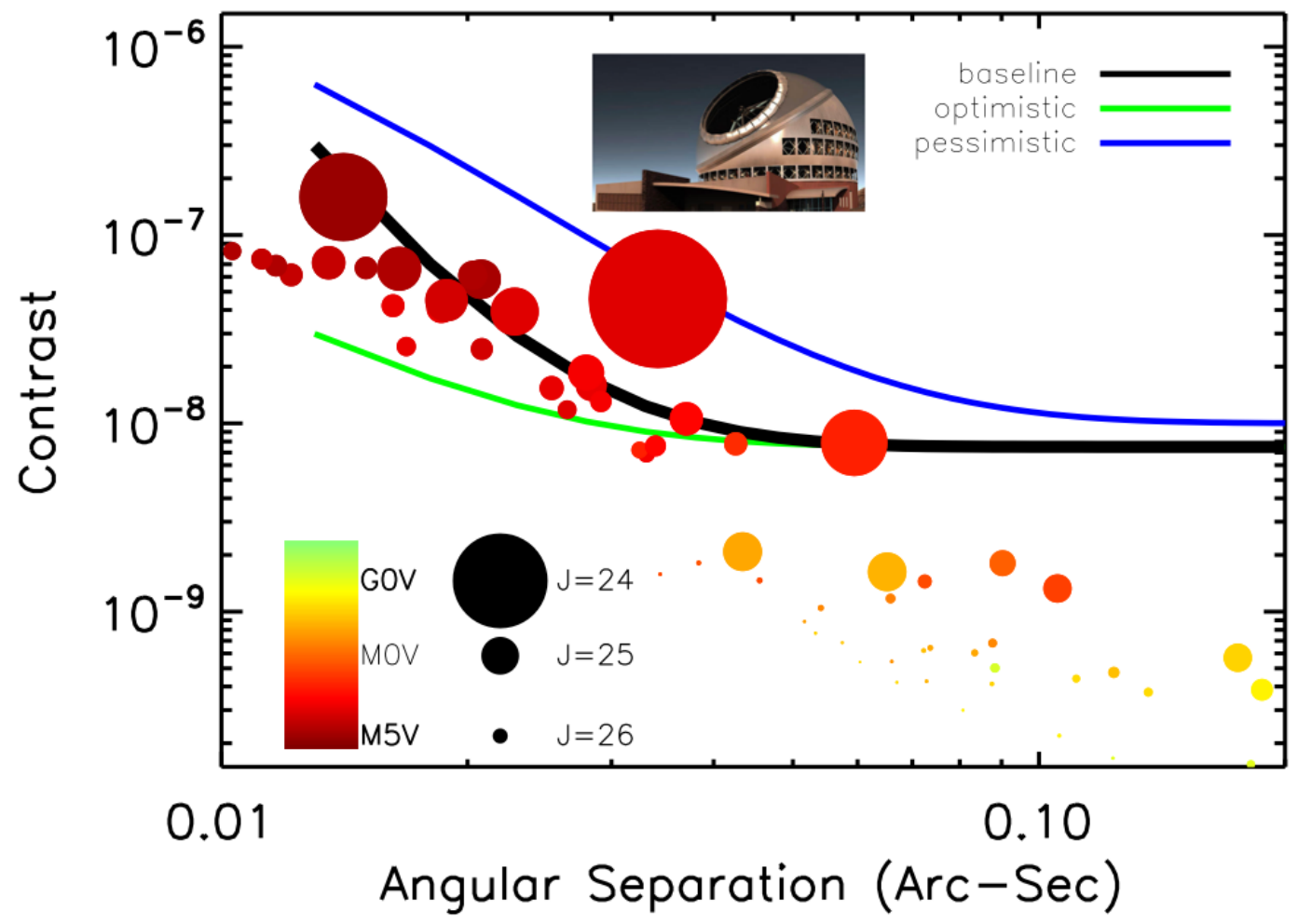

Figure 10. Contrast curve in $J$-band for a successor instrument to SCExAO put on the TMT - the Planetary Systems Imager (PSI) - using three different assumptions about its performance at small angular separations. Systems like SCExAO help mature wavefront sensing/control methods and coronagraphy needed to achieve these performances. Under optimistic performance scenarios $\left(\sim 10^{-6}\right.$ raw contrast and $\sim 3 \times 10^{-8}$ contrast after post-processing at $\left.\sim 1.5-2 \lambda / D\right)$, TMT/PSI could image rocky, Earth sized planets with an Earth-like insolation around about 20-25 nearby M stars.

On a slightly longer timescale, the key advance will be to replace the facility AO system - which uses a curvature wavefront sensor driving a DM with only 188 actuators - with a much faster $(2 \mathrm{kHz})$ and far higher-order DM (64x64 actuators) driven by a higher-performance wavefront sensor. Effectively, this two-stage SCExAO will allow the current wavefront sensor/DM loop to focus on speckle control/digging a much deeper dark hole and thus enabling a much larger planet discovery phase space. SCExAO is also maturing near-infrared wavefront sensing capabilities to better target optically faint stars and reduce non-common path aberrations.

\subsection{An Exoplanet Survey with SCExAO/CHARIS and the Path to Imaging Reflected-Light Planets with ELTs}

Our current plan is to carry out an exoplanet direct imaging survey using an upgraded SCExAO and the CHARIS integral field spectrograph once the system has achieved significantly deeper contrasts than demonstrated by GPI or SPHERE (e.g. $10^{-6}$ at $0.2^{\prime \prime}, 10^{-7}$ at wide separations). SCExAO performs substantially better in good seeing conditions. Therefore, one option is to conduct the survey in queue mode with other programs, for instance the current InfraRed Doppler (IRD) instrument conducting a near-IR precision radial-velocity survey of the nearest low-mass stars.

Current, blind surveys with GPI and SPHERE have resulted in a small yield of new exoplanet and low-mass brown dwarf discoveries, which limits the sample of companions with diverse properties, and thus prevents us from better mapping out the atmospheric evolution of supermassive jovian planets. However, target selection could be 
modified to substantially increase yields. For instance, targeted searches could prioritize stars showing evidence for a gravitational pull from an unseen substellar companion. The Hipparcos-GAIA Catalog of Accelerations $(\text { HGCA })^{50}$ provides a current master list of stars showing clear dynamical evidence for stellar to substellar companions. Upcoming GAIA data releases will identify more weakly accelerating stars hosting lower-mass planets. From a limited retargeting of bright, nearby HGCA stars we have already identified at least one new substellar companion using SCExAO/CHARIS. Focusing on the sample on stars already showing evidence for companions will prevent us from deriving strong, unbiased statistics on exoplanet frequency in exchange for a much higher yield of detections to better constrain planet atmosphere evolution.

Instruments like SCExAO as well as other upcoming systems like the Keck Planet Imager and Characterizer (KPIC) and MagAO-X mature key wavefront sensing and control technology that will be implemented on ELTs to directly image rocky planets in reflected light around the nearest stars. Habitable zone Earth-sized planets have contrasts of $\sim 10^{-7}-10^{-8}$ but lie at angular separations of $\sim 0.01$ " -0.1 " around $\mathrm{M}$ stars at $\sim 5 p c$ or $\sim 1-5$ $\lambda / \mathrm{D}$ for facilities like the Thirty Meter Telescope (TMT) and Giant Magellan Telescope (GMT) (Figure 10).

Key contributors to the wavefront error budget and thus limiting raw contrast at small angular separations include errors due to optical path length differences (between that of the wavefront sensor and science instrument wavelengths), AO servo lag and wavefront sensor noise, and scintillation (conversion of phase errors to amplitude modulation) which are predicted to operate at the $10^{-3}-10^{-4}, 10^{-5}$, and $10^{-6}$ contrast levels, respectively within $3-5 \lambda / \mathrm{D} .{ }^{51}$ Advances being matured on SCExAO address each of these terms in the error budget. For instance, focal-plane wavefront sensing/control being matured with SCExAO can help reduce optical path length errors; predictive control reduces the AO servo lag contribution.

Contrast curves in Figure 10 depict a range of performances for the Planetary Systems Imager - coarsely conceived as a successor to systems like SCExAO - on TMT depending on the system's efficacy with reducing each of these errors assuming a factor of $\sim 30$ for speckle suppression from post-processing. In the pessimistic case where substantial non-common path and servo lag/wavefront sensor noise contributions remain, TMT/PSI would detect perhaps one Earth-analogue around a nearby low-mass star but many more super Earths and warm Neptunes. For an optimistic case where TMT/PSI is able to substantially eliminate non-common path, servo lag, and wavefront sensor noise contributions, Earth-sized habitable zone planets could be detectable around nearly two-dozen M stars.

\section{ACKNOWLEDGMENTS}

T.C. is supported by a NASA Senior Postdoctoral Fellowship and NASA/Keck grant LK-2663-948181. We emphasize the pivotal cultural role and reverence that the summit of Maunakea has always had within the Hawaiian community. We are most fortunate to conduct scientific observations from this mountain.

\section{REFERENCES}

[1] Marois, C., Macintosh, B., Barman, T., Zuckerman, B., Song, I., Patience, J., Lafrenière, D., and Doyon, R., "Direct Imaging of Multiple Planets Orbiting the Star HR 8799," Science 322, 1348- (Nov. 2008).

[2] Marois, C., Zuckerman, B., Konopacky, Q. M., Macintosh, B., and Barman, T., "Images of a fourth planet orbiting HR 8799," Nature 468, 1080-1083 (Dec 2010).

[3] Lagrange, A.-M., Bonnefoy, M., Chauvin, G., Apai, D., Ehrenreich, D., Boccaletti, A., Gratadour, D., Rouan, D., Mouillet, D., Lacour, S., and Kasper, M., "A Giant Planet Imaged in the Disk of the Young Star $\beta$ Pictoris," Science 329, 57 (July 2010).

[4] Rameau, J., Chauvin, G., Lagrange, A. M., Meshkat, T., Boccaletti, A., Quanz, S. P., Currie, T., Mawet, D., Girard, J. H., Bonnefoy, M., and Kenworthy, M., "Confirmation of the Planet around HD 95086 by Direct Imaging," Astrophysical Journal Letters 779, L26 (Dec 2013).

[5] Currie, T., Daemgen, S., Debes, J., Lafreniere, D., Itoh, Y., Jayawardhana, R., Ratzka, T., and Correia, S., "Direct Imaging and Spectroscopy of a Candidate Companion Below/Near the Deuterium-burning Limit in the Young Binary Star System, ROXs 42B," Astrophysical Journal Letters 780, L30 (Jan 2014). 
[6] Currie, T., Burrows, A., Itoh, Y., Matsumura, S., Fukagawa, M., Apai, D., Madhusudhan, N., Hinz, P. M., Rodigas, T. J., Kasper, M., Pyo, T.-S., and Ogino, S., "A Combined Subaru/VLT/MMT 1-5 $\mu$ m Study of Planets Orbiting HR 8799: Implications for Atmospheric Properties, Masses, and Formation," Astrophysical Journal 729, 128 (Mar. 2011).

[7] Galicher, R., Marois, C., Macintosh, B., Barman, T., and Konopacky, Q., "M-band Imaging of the HR 8799 Planetary System Using an Innovative LOCI-based Background Subtraction Technique," Astrophysical Journal Letters 739, L41 (Oct 2011).

[8] Barman, T. S., Macintosh, B., Konopacky, Q. M., and Marois, C., "Clouds and Chemistry in the Atmosphere of Extrasolar Planet HR8799b," Astrophysical Journal 733, 65 (May 2011).

[9] Brandt, T. D., Kuzuhara, M., McElwain, M. W., Schlieder, J. E., Wisniewski, J. P., Turner, E. L., Carson, J., Matsuo, T., Biller, B., Bonnefoy, M., Dressing, C., Janson, M., Knapp, G. R., Moro-Martín, A., Thalmann, C., Kudo, T., Kusakabe, N., Hashimoto, J., Abe, L., Brandner, W., Currie, T., Egner, S., Feldt, M., Golota, T., Goto, M., Grady, C. A., Guyon, O., Hayano, Y., Hayashi, M., Hayashi, S., Henning, T., Hodapp, K. W., Ishii, M., Iye, M., Kand ori, R., Kwon, J., Mede, K., Miyama, S., Morino, J. I., Nishimura, T., Pyo, T. S., Serabyn, E., Suenaga, T., Suto, H., Suzuki, R., Takami, M., Takahashi, Y., Takato, N., Terada, H., Tomono, D., Watanabe, M., Yamada, T., Takami, H., Usuda, T., and Tamura, M., "The Moving Group Targets of the SEEDS High-contrast Imaging Survey of Exoplanets and Disks: Results and Observations from the First Three Years," Astrophysical Journal 786, 1 (May 2014).

[10] Brandt, T. D., McElwain, M. W., Turner, E. L., Mede, K., Spiegel, D. S., Kuzuhara, M., Schlieder, J. E., Wisniewski, J. P., Abe, L., Biller, B., Brandner, W., Carson, J., Currie, T., Egner, S., Feldt, M., Golota, T., Goto, M., Grady, C. A., Guyon, O., Hashimoto, J., Hayano, Y., Hayashi, M., Hayashi, S., Henning, T., Hodapp, K. W., Inutsuka, S., Ishii, M., Iye, M., Janson, M., Kand ori, R., Knapp, G. R., Kudo, T., Kusakabe, N., Kwon, J., Matsuo, T., Miyama, S., Morino, J. I., Moro-Martín, A., Nishimura, T., Pyo, T. S., Serabyn, E., Suto, H., Suzuki, R., Takami, M., Takato, N., Terada, H., Thalmann, C., Tomono, D., Watanabe, M., Yamada, T., Takami, H., Usuda, T., and Tamura, M., "A Statistical Analysis of SEEDS and Other High-contrast Exoplanet Surveys: Massive Planets or Low-mass Brown Dwarfs?," The Astrophysical Journal 794, 159 (Oct 2014).

[11] Macintosh, B., Graham, J. R., Ingraham, P., Konopacky, Q., Marois, C., Perrin, M., Poyneer, L., Bauman, B., Barman, T., Burrows, A. S., Cardwell, A., Chilcote, J., De Rosa, R. J., Dillon, D., Doyon, R., Dunn, J., Erikson, D., Fitzgerald, M. P., Gavel, D., Goodsell, S., Hartung, M., Hibon, P., Kalas, P., Larkin, J., Maire, J., Marchis, F., Marley, M. S., McBride, J., Millar-Blanchaer, M., Morzinski, K., Norton, A., Oppenheimer, B. R., Palmer, D., Patience, J., Pueyo, L., Rantakyro, F., Sadakuni, N., Saddlemyer, L., Savransky, D., Serio, A., Soummer, R., Sivaramakrishnan, A., Song, I., Thomas, S., Wallace, J. K., Wiktorowicz, S., and Wolff, S., "First light of the Gemini Planet Imager," Proceedings of the National Academy of Science 111, 12661-12666 (Sep 2014).

[12] Vigan, A., Gry, C., Salter, G., Mesa, D., Homeier, D., Moutou, C., and Allard, F., "High-contrast imaging of Sirius A with VLT/SPHERE: looking for giant planets down to one astronomical unit," Monthly Notices of the Royal Astronomical Society 454, 129-143 (Nov 2015).

[13] Macintosh, B., Graham, J. R., Barman, T., De Rosa, R. J., Konopacky, Q., Marley, M. S., Marois, C., Nielsen, E. L., Pueyo, L., Rajan, A., Rameau, J., Saumon, D., Wang, J. J., Patience, J., Ammons, M., Arriaga, P., Artigau, E., Beckwith, S., Brewster, J., Bruzzone, S., Bulger, J., Burningham, B., Burrows, A. S., Chen, C., Chiang, E., Chilcote, J. K., Dawson, R. I., Dong, R., Doyon, R., Draper, Z. H., Duchêne, G., Esposito, T. M., Fabrycky, D., Fitzgerald, M. P., Follette, K. B., Fortney, J. J., Gerard, B., Goodsell, S., Greenbaum, A. Z., Hibon, P., Hinkley, S., Cotten, T. H., Hung, L. W., Ingraham, P., Johnson-Groh, M., Kalas, P., Lafreniere, D., Larkin, J. E., Lee, J., Line, M., Long, D., Maire, J., Marchis, F., Matthews, B. C., Max, C. E., Metchev, S., Millar-Blanchaer, M. A., Mittal, T., Morley, C. V., Morzinski, K. M., Murray-Clay, R., Oppenheimer, R., Palmer, D. W., Patel, R., Perrin, M. D., Poyneer, L. A., Rafikov, R. R., Rantakyrö, F. T., Rice, E. L., Rojo, P., Rudy, A. R., Ruffio, J. B., Ruiz, M. T., Sadakuni, N., Saddlemyer, L., Salama, M., Savransky, D., Schneider, A. C., Sivaramakrishnan, A., Song, I., Soummer, R., Thomas, S., Vasisht, G., Wallace, J. K., Ward- Duong, K., Wiktorowicz, S. J., Wolff, S. G., and Zuckerman, B., "Discovery and spectroscopy of the young jovian planet 51 Eri b with the Gemini Planet Imager," Science 350, 64-67 (Oct. 2015). 
[14] Chauvin, G., Desidera, S., Lagrange, A. M., Vigan, A., Gratton, R., Langlois, M., Bonnefoy, M., Beuzit, J. L., Feldt, M., Mouillet, D., Meyer, M., Cheetham, A., Biller, B., Boccaletti, A., D'Orazi, V., Galicher, R., Hagelberg, J., Maire, A. L., Mesa, D., Olofsson, J., Samland, M., Schmidt, T. O. B., Sissa, E., Bonavita, M., Charnay, B., Cudel, M., Daemgen, S., Delorme, P., Janin-Potiron, P., Janson, M., Keppler, M., Le Coroller, H., Ligi, R., Marleau, G. D., Messina, S., Mollière, P., Mordasini, C., Müller, A., Peretti, S., Perrot, C., Rodet, L., Rouan, D., Zurlo, A., Dominik, C., Henning, T., Menard, F., Schmid, H. M., Turatto, M., Udry, S., Vakili, F., Abe, L., Antichi, J., Baruffolo, A., Baudoz, P., Baudrand, J., Blanchard, P., Bazzon, A., Buey, T., Carbillet, M., Carle, M., Charton, J., Cascone, E., Claudi, R., Costille, A., Deboulbe, A., De Caprio, V., Dohlen, K., Fantinel, D., Feautrier, P., Fusco, T., Gigan, P., Giro, E., Gisler, D., Gluck, L., Hubin, N., Hugot, E., Jaquet, M., Kasper, M., Madec, F., Magnard, Y., Martinez, P., Maurel, D., Le Mignant, D., Möller-Nilsson, O., Llored, M., Moulin, T., Origné, A., Pavlov, A., Perret, D., Petit, C., Pragt, J., Puget, P., Rabou, P., Ramos, J., Rigal, R., Rochat, S., Roelfsema, R., Rousset, G., Roux, A., Salasnich, B., Sauvage, J. F., Sevin, A., Soenke, C., Stadler, E., Suarez, M., Weber, L., Wildi, F., Antoniucci, S., Augereau, J. C., Baudino, J. L., Brandner, W., Engler, N., Girard, J., Gry, C., Kral, Q., Kopytova, T., Lagadec, E., Milli, J., Moutou, C., Schlieder, J., Szulágyi, J., Thalmann, C., and Wahhaj, Z., "Discovery of a warm, dusty giant planet around HIP 65426," Astronomy 83 Astrophysics 605, L9 (Sep 2017).

[15] Currie, T., Cloutier, R., Brittain, S., Grady, C., Burrows, A., Muto, T., Kenyon, S. J., and Kuchner, M. J., "Resolving the HD 100546 Protoplanetary System with the Gemini Planet Imager: Evidence for Multiple Forming, Accreting Planets," Astrophysical Journal Letters 814, L27 (Dec 2015).

[16] Milli, J., Hibon, P., Christiaens, V., Choquet, É., Bonnefoy, M., Kennedy, G. M., Wyatt, M. C., Absil, O., Gómez González, C. A., del Burgo, C., Matrà, L., Augereau, J. C., Boccaletti, A., Delacroix, C., Ertel, S., Dent, W. R. F., Forsberg, P., Fusco, T., Girard, J. H., Habraken, S., Huby, E., Karlsson, M., Lagrange, A. M., Mawet, D., Mouillet, D., Perrin, M., Pinte, C., Pueyo, L., Reyes, C., Soummer, R., Surdej, J., Tarricq, Y., and Wahhaj, Z., "Discovery of a low-mass companion inside the debris ring surrounding the F5V star HD 206893," Astronomy 83 Astrophysics 597, L2 (Jan 2017).

[17] Keppler, M., Benisty, M., Müller, A., Henning, T., van Boekel, R., Cantalloube, F., Ginski, C., van Holstein, R. G., Maire, A. L., Pohl, A., Samland, M., Avenhaus, H., Baudino, J. L., Boccaletti, A., de Boer, J., Bonnefoy, M., Chauvin, G., Desidera, S., Langlois, M., Lazzoni, C., Marleau, G. D., Mordasini, C., Pawellek, N., Stolker, T., Vigan, A., Zurlo, A., Birnstiel, T., Brandner, W., Feldt, M., Flock, M., Girard, J., Gratton, R., Hagelberg, J., Isella, A., Janson, M., Juhasz, A., Kemmer, J., Kral, Q., Lagrange, A. M., Launhardt, R., Matter, A., Ménard, F., Milli, J., Mollière, P., Olofsson, J., Pérez, L., Pinilla, P., Pinte, C., Quanz, S. P., Schmidt, T., Udry, S., Wahhaj, Z., Williams, J. P., Buenzli, E., Cudel, M., Dominik, C., Galicher, R., Kasper, M., Lannier, J., Mesa, D., Mouillet, D., Peretti, S., Perrot, C., Salter, G., Sissa, E., Wildi, F., Abe, L., Antichi, J., Augereau, J. C., Baruffolo, A., Baudoz, P., Bazzon, A., Beuzit, J. L., Blanchard, P., Brems, S. S., Buey, T., De Caprio, V., Carbillet, M., Carle, M., Cascone, E., Cheetham, A., Claudi, R., Costille, A., Delboulbé, A., Dohlen, K., Fantinel, D., Feautrier, P., Fusco, T., Giro, E., Gluck, L., Gry, C., Hubin, N., Hugot, E., Jaquet, M., Le Mignant, D., Llored, M., Madec, F., Magnard, Y., Martinez, P., Maurel, D., Meyer, M., Möller-Nilsson, O., Moulin, T., Mugnier, L., Origné, A., Pavlov, A., Perret, D., Petit, C., Pragt, J., Puget, P., Rabou, P., Ramos, J., Rigal, F., Rochat, S., Roelfsema, R., Rousset, G., Roux, A., Salasnich, B., Sauvage, J. F., Sevin, A., Soenke, C., Stadler, E., Suarez, M., Turatto, M., and Weber, L., "Discovery of a planetary-mass companion within the gap of the transition disk around PDS 70," Astronomy \& Astrophysics 617, A44 (Sep 2018).

[18] Bonnefoy, M., Zurlo, A., Baudino, J. L., Lucas, P., Mesa, D., Maire, A. L., Vigan, A., Galicher, R., Homeier, D., Marocco, F., Gratton, R., Chauvin, G., Allard, F., Desidera, S., Kasper, M., Moutou, C., Lagrange, A. M., Antichi, J., Baruffolo, A., Baudrand, J., Beuzit, J. L., Boccaletti, A., Cantalloube, F., Carbillet, M., Charton, J., Claudi, R. U., Costille, A., Dohlen, K., Dominik, C., Fantinel, D., Feautrier, P., Feldt, M., Fusco, T., Gigan, P., Girard, J. H., Gluck, L., Gry, C., Henning, T., Janson, M., Langlois, M., Madec, F., Magnard, Y., Maurel, D., Mawet, D., Meyer, M. R., Milli, J., Moeller-Nilsson, O., Mouillet, D., Pavlov, A., Perret, D., Pujet, P., Quanz, S. P., Rochat, S., Rousset, G., Roux, A., Salasnich, B., Salter, G., Sauvage, J. F., Schmid, H. M., Sevin, A., Soenke, C., Stadler, E., Turatto, M., Udry, S., Vakili, F., Wahhaj, Z., and Wildi, F., "First light of the VLT planet finder SPHERE. IV. Physical and chemical properties of the planets around HR8799," Astronomy 83 Astrophysics 587, A58 (Mar 2016). 
[19] Chilcote, J., Pueyo, L., De Rosa, R. J., Vargas, J., Macintosh, B., Bailey, V. P., Barman, T., Bauman, B., Bruzzone, S., Bulger, J., Burrows, A. S., Cardwell, A., Chen, C. H., Cotten, T., Dillon, D., Doyon, R., Draper, Z. H., Duchêne, G., Dunn, J., Erikson, D., Fitzgerald, M. P., Follette, K. B., Gavel, D., Goodsell, S. J., Graham, J. R., Greenbaum, A. r. Z., Hartung, M., Hibon, P., Hung, L.-W., Ingraham, P., Kalas, P., Konopacky, Q., Larkin, J. E., Maire, J., Marchis, F., Marley, M. S., Marois, C., Metchev, S., MillarBlanchaer, M. A., Morzinski, K. M., Nielsen, E. L., Norton, A., Oppenheimer, R., Palmer, D., Patience, J., Perrin, M., Poyneer, L., Rajan, A., Rameau, J., Rantakyrö, F. T., Sadakuni, N., Saddlemyer, L., Savransky, D., Schneider, A. C., Serio, A., Sivaramakrishnan, A., Song, I., Soummer, R., Thomas, S., Wallace, J. K., Wang, J. J., Ward-Duong, K., Wiktorowicz, S., and Wolff, S., "1-2.4 $\mu \mathrm{m}$ Near-IR Spectrum of the Giant Planet $\beta$ Pictoris b Obtained with the Gemini Planet Imager," Astronomical Journal 153, 182 (Apr 2017 ).

[20] Rajan, A., Rameau, J., De Rosa, R. J., Marley, M. S., Graham, J. R., Macintosh, B., Marois, C., Morley, C., Patience, J., Pueyo, L., Saumon, D., Ward-Duong, K., Ammons, S. M., Arriaga, P., Bailey, V. P., Barman, T., Bulger, J., Burrows, A. S., Chilcote, J., Cotten, T., Czekala, I., Doyon, R., Duchêne, G., Esposito, T. M., Fitzgerald, M. P., Follette, K. B., Fortney, J. J., Goodsell, S. J., Greenbaum, A. r. Z., Hibon, P., Hung, L.-W., Ingraham, P., Johnson-Groh, M., Kalas, P., Konopacky, Q., Lafrenière, D., Larkin, J. E., Maire, J., Marchis, F., Metchev, S., Millar-Blanchaer, M. A., Morzinski, K. M., Nielsen, E. L., Oppenheimer, R., Palmer, D., Patel, R. I., Perrin, M., Poyneer, L., Rantakyrö, F. T., Ruffio, J.-B., Savransky, D., Schneider, A. C., Sivaramakrishnan, A., Song, I., Soummer, R., Thomas, S., Vasisht, G., Wallace, J. K., Wang, J. J., Wiktorowicz, S., and Wolff, S., "Characterizing 51 Eri b from 1 to $5 \mu \mathrm{m}$ : A Partly Cloudy Exoplanet," Astronomical Journal 154, 10 (Jul 2017).

[21] Nielsen, E. L., De Rosa, R. J., Macintosh, B., Wang, J. J., Ruffio, J.-B., Chiang, E., Marley, M. S., Saumon, D., Savransky, D., Ammons, S. M., Bailey, V. P., Barman, T., Blain, C., Bulger, J., Burrows, A., Chilcote, J., Cotten, T., Czekala, I., Doyon, R., Duchêne, G., Esposito, T. M., Fabrycky, D., Fitzgerald, M. P., Follette, K. B., Fortney, J. J., Gerard, B. L., Goodsell, S. J., Graham, J. R., Greenbaum, A. Z., Hibon, P., Hinkley, S., Hirsch, L. A., Hom, J., Hung, L.-W., Dawson, R. I., Ingraham, P., Kalas, P., Konopacky, Q., Larkin, J. E., Lee, E. J., Lin, J. W., Maire, J., Marchis, F., Marois, C., Metchev, S., Millar-Blanchaer, M. A., Morzinski, K. M., Oppenheimer, R., Palmer, D., Patience, J., Perrin, M., Poyneer, L., Pueyo, L., Rafikov, R. R., Rajan, A., Rameau, J., Rantakyrö, F. T., Ren, B., Schneider, A. C., Sivaramakrishnan, A., Song, I., Soummer, R., Tallis, M., Thomas, S., Ward-Duong, K., and Wolff, S., "The Gemini Planet Imager Exoplanet Survey: Giant Planet and Brown Dwarf Demographics from 10 to 100 au," Astronomical Journal 158, 13 (Jul 2019).

[22] Fernandes, R. B., Mulders, G. D., Pascucci, I., Mordasini, C., and Emsenhuber, A., "Hints for a Turnover at the Snow Line in the Giant Planet Occurrence Rate," Astrophysical Journal 874, 81 (Mar 2019 ).

[23] Jovanovic, N., Martinache, F., Guyon, O., Clergeon, C., Singh, G., Kudo, T., Garrel, V., Newman, K., Doughty, D., Lozi, J., Males, J., Minowa, Y., Hayano, Y., Takato, N., Morino, J., Kuhn, J., Serabyn, E., Norris, B., Tuthill, P., Schworer, G., Stewart, P., Close, L., Huby, E., Perrin, G., Lacour, S., Gauchet, L., Vievard, S., Murakami, N., Oshiyama, F., Baba, N., Matsuo, T., Nishikawa, J., Tamura, M., Lai, O., Marchis, F., Duchene, G., Kotani, T., and Woillez, J., "The Subaru Coronagraphic Extreme Adaptive Optics System: Enabling High-Contrast Imaging on Solar-System Scales," Publications of the Astronomical Society of the Pacific 127, 890 (Sep 2015).

[24] Brandt, T. D., Rizzo, M., Groff, T., Chilcote, J., Greco, J. P., Kasdin, N. J., Limbach, M. A., Galvin, M., Loomis, C., Knapp, G., McElwain, M. W., Jovanovic, N., Currie, T., Mede, K., Tamura, M., Takato, N., and Hayashi, M., "Data reduction pipeline for the CHARIS integral-field spectrograph I: detector readout calibration and data cube extraction," Journal of Astronomical Telescopes, Instruments, and Systems 3, 048002 (Oct 2017).

[25] Currie, T., Brandt, T. D., Uyama, T., Nielsen, E. L., Blunt, S., Guyon, O., Tamura, M., Marois, C., Mede, K., Kuzuhara, M., Groff, T. D., Jovanovic, N., Kasdin, N. J., Lozi, J., Hodapp, K., Chilcote, J., Carson, J., Martinache, F., Goebel, S., Grady, C., McElwain, M., Akiyama, E., Asensio-Torres, R., Hayashi, M., Janson, M., Knapp, G. R., Kwon, J., Nishikawa, J., Oh, D., Schlieder, J., Serabyn, E., Sitko, M., and Skaf, N., "SCExAO/CHARIS Near-infrared Direct Imaging, Spectroscopy, and Forward-Modeling of $\kappa$ And b: A Likely Young, Low-gravity Superjovian Companion," Astronomical Journal 156, 291 (Dec 2018). 
[26] Currie, T., Kasdin, N. J., Groff, T. D., Lozi, J., Jovanovic, N., Guyon, O., Brand t, T., Martinache, F., Chilcote, J., Skaf, N., Kuhn, J., Pathak, P., and Kudo, T., "Laboratory and On-sky Validation of the Shaped Pupil Coronagraph's Sensitivity to Low-order Aberrations With Active Wavefront Control," Publications of the Astronomical Society of the Pacific 130, 044505 (Apr 2018).

[27] Wang, J. J., Ruffio, J.-B., De Rosa, R. J., Aguilar, J., Wolff, S. G., and Pueyo, L., "pyKLIP: PSF Subtraction for Exoplanets and Disks," (Jun 2015).

[28] Marois, C., Correia, C., Galicher, R., Ingraham, P., Macintosh, B., Currie, T., and De Rosa, R., "GPI PSF subtraction with TLOCI: the next evolution in exoplanet/disk high-contrast imaging," in [Proc. SPIE.], Society of Photo-Optical Instrumentation Engineers (SPIE) Conference Series 9148, 91480U (Jul 2014).

[29] Gerard, B. L., Marois, C., Currie, T., Brandt, T., Chilcote, J. K., Draper, Z. H., Groff, T., Guyon, O., Hayashi, M., Jovanovic, N., Knapp, G. R., Kudo, T., Kwon, J., Lozi, J., Martinache, F., McElwain, M., Tamura, M., and Uyama, T., "A Chromaticity Analysis and PSF Subtraction Techniques for SCExAO/CHARIS Data," Astronomical Journal 158, 36 (Jul 2019).

[30] Beuzit, J. L., Vigan, A., Mouillet, D., Dohlen, K., Gratton, R., Boccaletti, A., Sauvage, J. F., Schmid, H. M., Langlois, M., Petit, C., Baruffolo, A., Feldt, M., Milli, J., Wahhaj, Z., Abe, L., Anselmi, U., Antichi, J., Barette, R., Baudrand, J., Baudoz, P., Bazzon, A., Bernardi, P., Blanchard, P., Brast, R., Bruno, P., Buey, T., Carbillet, M., Carle, M., Cascone, E., Chapron, F., Chauvin, G., Charton, J., Claudi, R., Costille, A., De Caprio, V., Delboulbé, A., Desidera, S., Dominik, C., Downing, M., Dupuis, O., Fabron, C., Fantinel, D., Farisato, G., Feautrier, P., Fedrigo, E., Fusco, T., Gigan, P., Ginski, C., Girard, J., Giro, E., Gisler, D., Gluck, L., Gry, C., Henning, T., Hubin, N., Hugot, E., Incorvaia, S., Jaquet, M., Kasper, M., Lagadec, E., Lagrange, A. M., Le Coroller, H., Le Mignant, D., Le Ruyet, B., Lessio, G., Lizon, J. L., Llored, M., Lundin, L., Madec, F., Magnard, Y., Marteaud, M., Martinez, P., Maurel, D., Ménard, F., Mesa, D., Möller-Nilsson, O., Moulin, T., Moutou, C., Origné, A., Parisot, J., Pavlov, A., Perret, D., Pragt, J., Puget, P., Rabou, P., Ramos, J., Reess, J. M., Rigal, F., Rochat, S., Roelfsema, R., Rousset, G., Roux, A., Saisse, M., Salasnich, B., Santambrogio, E., Scuderi, S., Segransan, D., Sevin, A., Siebenmorgen, R., Soenke, C., Stadler, E., Suarez, M., Tiphène, D., Turatto, M., Udry, S., Vakili, F., Waters, L. B. F. M., Weber, L., Wildi, F., Zins, G., and Zurlo, A., "SPHERE: the exoplanet imager for the Very Large Telescope," arXiv e-prints , arXiv:1902.04080 (Feb 2019).

[31] Marois, C., Lafrenière, D., Doyon, R., Macintosh, B., and Nadeau, D., "Angular Differential Imaging: A Powerful High-Contrast Imaging Technique," Astrophysical Journal 641, 556-564 (Apr 2006).

[32] Currie, T., Debes, J., Rodigas, T. J., Burrows, A., Itoh, Y., Fukagawa, M., Kenyon, S. J., Kuchner, M., and Matsumura, S., "Direct Imaging Confirmation and Characterization of a Dust-enshrouded Candidate Exoplanet Orbiting Fomalhaut," Astrophysical Journal Letters 760, L32 (Dec 2012).

[33] Currie, T., Marois, C., Cieza, L., Mulders, G. D., Lawson, K., Caceres, C., Rodriguez-Ruiz, D., Wisniewski, J., Guyon, O., Brandt, T. D., Kasdin, N. J., Groff, T. D., Lozi, J., Chilcote, J., Hodapp, K., Jovanovic, N., Martinache, F., Skaf, N., Lyra, W., Tamura, M., Asensio-Torres, R., Dong, R., Grady, C., Gerard, B., Fukagawa, M., Hand, D., Hayashi, M., Henning, T., Kudo, T., Kuzuhara, M., Kwon, J., McElwain, M. W., and Uyama, T., "No Clear, Direct Evidence for Multiple Protoplanets Orbiting LkCa 15: LkCa 15 bcd are Likely Inner Disk Signals," Astrophysical Journal Letters 877, L3 (May 2019).

[34] Goebel, S., Currie, T., Guyon, O., Brand t, T. D., Groff, T. D., Jovanovic, N., Kasdin, N. J., Lozi, J., Hodapp, K., Martinache, F., Grady, C., Hayashi, M., Kwon, J., McElwain, M. W., Yang, Y., and Tamura, M., "SCExAO/CHARIS Near-IR High-contrast Imaging and Integral Field Spectroscopy of the HIP 79977 Debris Disk," Astronomical Journal 156, 279 (Dec 2018).

[35] Asensio-Torres, R., Currie, T., Janson, M., Desidera, S., Kuzuhara, M., Hodapp, K., Brandt, T. D., Guyon, O., Lozi, J., Groff, T., Kasdin, J., Chilcote, J., Jovanovic, N., Martinache, F., Sitko, M., Serabyn, E., Wagner, K., Akiyama, E., Kwon, J., Uyama, T., Yang, Y., Nakagawa, T., Hayashi, M., McElwain, M., Kudo, T., Henning, T., and Tamura, M., "Isochronal age-mass discrepancy of young stars: SCExAO/CHARIS integral field spectroscopy of the HIP 79124 triple system," Astronomy \& Astrophysics 622, A42 (Feb 2019).

[36] Rich, E. A., Wisniewski, J. P., Currie, T., Fukagawa, M., Grady, C. A., Sitko, M. L., Pikhartova, M., Hashimoto, J., Abe, L., Brand ner, W., Brandt, T. D., Carson, J. C., Chilcote, J., Dong, R., Feldt, M., Goto, M., Groff, T., Guyon, O., Hayano, Y., Hayashi, M., Hayashi, S. S., Henning, T., Hodapp, K. W., Ishii, M., Iye, M., Janson, M., Jovanovic, N., Kand ori, R., Kasdin, J., Knapp, G. R., Kudo, T., 
Kusakabe, N., Kuzuhara, M., Kwon, J., Lozi, J., Martinache, F., Matsuo, T., Mayama, S., McElwain, M. W., Miyama, S., Morino, J.-I., Moro-Martin, A., Nakagawa, T., Nishimura, T., Pyo, T.-S., Serabyn, E., Suto, H., Russel, R. W., Suzuki, R., Takami, M., Takato, N., Terada, H., Thalmann, C., Turner, E. L., Uyama, T., Wagner, K. R., Watanabe, M., Yamada, T., Takami, H., Usuda, T., and Tamura, M., "Multiepoch Direct Imaging and Time-variable Scattered Light Morphology of the HD 163296 Protoplanetary Disk," Astrophysical Journal 875, 38 (Apr 2019).

[37] Carson, J., Thalmann, C., Janson, M., Kozakis, T., Bonnefoy, M., Biller, B., Schlieder, J., Currie, T., McElwain, M., Goto, M., Henning, T., Brandner, W., Feldt, M., Kandori, R., Kuzuhara, M., Stevens, L., Wong, P., Gainey, K., Fukagawa, M., Kuwada, Y., Brand t, T., Kwon, J., Abe, L., Egner, S., Grady, C., Guyon, O., Hashimoto, J., Hayano, Y., Hayashi, M., Hayashi, S., Hodapp, K., Ishii, M., Iye, M., Knapp, G., Kudo, T., Kusakabe, N., Matsuo, T., Miyama, S., Morino, J., Moro-Martin, A., Nishimura, T., Pyo, T., Serabyn, E., Suto, H., Suzuki, R., Takami, M., Takato, N., Terada, H., Tomono, D., Turner, E., Watanabe, M., Wisniewski, J., Yamada, T., Takami, H., Usuda, T., and Tamura, M., "Direct Imaging Discovery of a "Super-Jupiter" around the Late B-type Star $\kappa$ And," Astrophysical Journal Letters 763, L32 (Feb 2013).

[38] Hinkley, S., Pueyo, L., Faherty, J. K., Oppenheimer, B. R., Mamajek, E. E., Kraus, A. L., Rice, E. L., Ireland, M. J., David, T., Hillenbrand, L. A., Vasisht, G., Cady, E., Brenner, D., Veicht, A., Nilsson, R., Zimmerman, N., Parry, I. R., Beichman, C., Dekany, R., Roberts, J. E., Roberts, Lewis C., J., Baranec, C., Crepp, J. R., Burruss, R., Wallace, J. K., King, D., Zhai, C., Lockhart, T., Shao, M., Soummer, R., Sivaramakrishnan, A., and Wilson, L. A., "The $\kappa$ Andromedae System: New Constraints on the Companion Mass, System Age, and Further Multiplicity," Astrophysical Journal 779, 153 (Dec 2013).

[39] Jones, J., White, R. J., Quinn, S., Ireland, M., Boyajian, T., Schaefer, G., and Baines, E. K., "The Age of the Directly Imaged Planet Host Star $\kappa$ Andromedae Determined from Interferometric Observations," Astrophysical Journal Letters 822, L3 (May 2016).

[40] Boss, A. P., "Giant planet formation by gravitational instability.," Science 276, 1836-1839 (Jan 1997).

[41] Espaillat, C., Calvet, N., D’Alessio, P., Hernández, J., Qi, C., Hartmann, L., Furlan, E., and Watson, D. M., "On the Diversity of the Taurus Transitional Disks: UX Tauri A and LkCa 15," Astrophysical Journal Letters 670, L135-L138 (Dec 2007).

[42] Andrews, S. M., Rosenfeld, K. A., Wilner, D. J., and Bremer, M., "A Closer Look at the LkCa 15 Protoplanetary Disk," Astrophysical Journal Letters 742, L5 (Nov 2011).

[43] Kraus, A. L. and Ireland, M. J., "LkCa 15: A Young Exoplanet Caught at Formation?," Astrophysical Journal 745, 5 (Jan 2012).

[44] Sallum, S., Follette, K. B., Eisner, J. A., Close, L. M., Hinz, P., Kratter, K., Males, J., Skemer, A., Macintosh, B., Tuthill, P., Bailey, V., Defrère, D., Morzinski, K., Rodigas, T., Spalding, E., Vaz, A., and Weinberger, A. J., "Accreting protoplanets in the LkCa 15 transition disk," Nature 527, 342-344 (Nov 2015).

[45] Gratton, R., Ligi, R., Sissa, E., Desidera, S., Mesa, D., Bonnefoy, M., Chauvin, G., Cheetham, A., Feldt, M., Lagrange, A. M., Langlois, M., Meyer, M., Vigan, A., Boccaletti, A., Janson, M., Lazzoni, C., Zurlo, A., De Boer, J., Henning, T., D’Orazi, V., Gluck, L., Madec, F., Jaquet, M., Baudoz, P., Fantinel, D., Pavlov, A., and Wildi, F., "Blobs, spiral arms, and a possible planet around HD 169142," Astronomy \&6 Astrophysics 623, A140 (Mar 2019).

[46] Lozi, J., Guyon, O., Jovanovic, N., Goebel, S., Pathak, P., Skaf, N., Sahoo, A., Norris, B., Martinache, F., N'Diaye, M., Mazin, B., Walter, A. B., Tuthill, P., Kudo, T., Kawahara, H., Kotani, T., Ireland, M., Cvetojevic, N., Huby, E., Lacour, S., Vievard, S., Groff, T. D., Chilcote, J. K., Kasdin, J., Knight, J., Snik, F., Doelman, D., Minowa, Y., Clergeon, C., Takato, N., Tamura, M., Currie, T., Takami, H., and Hayashi, M., "SCExAO, an instrument with a dual purpose: perform cutting-edge science and develop new technologies," in [Proc. SPIE.], Society of Photo-Optical Instrumentation Engineers (SPIE) Conference Series 10703, 1070359 (Jul 2018).

[47] Males, J. R., Close, L. M., Miller, K., Schatz, L., Doelman, D., Lumbres, J., Snik, F., Rodack, A., Knight, J., Van Gorkom, K., Long, J. D., Hedglen, A., Kautz, M., Jovanovic, N., Morzinski, K., Guyon, O., Douglas, E., Follette, K. B., Lozi, J., Bohlman, C., Durney, O., Gasho, V., Hinz, P., Ireland, M., Jean, M., Keller, C., Kenworthy, M., Mazin, B., Noenickx, J., Alfred, D., Perez, K., Sanchez, A., Sauve, C., Weinberger, 
A., and Conrad, A., "MagAO-X: project status and first laboratory results," in [Proc. SPIE.], Society of Photo-Optical Instrumentation Engineers (SPIE) Conference Series 10703, 1070309 (Jul 2018).

[48] Miller, K., Guyon, O., and Males, J., "Spatial linear dark field control: stabilizing deep contrast for exoplanet imaging using bright speckles," Journal of Astronomical Telescopes, Instruments, and Systems 3, 049002 (Oct 2017).

[49] Currie, T., Pluzhnik, E., Belikov, R., and Guyon, O., "Developing linear dark-field control for exoplanet direct imaging in the laboratory and on ground-based telescopes," in [Proc. SPIE.], Society of Photo-Optical Instrumentation Engineers (SPIE) Conference Series (2019).

[50] Brandt, T. D., "The Hipparcos-Gaia Catalog of Accelerations," Astrophysical Journal Supplements 239, 31 (Dec 2018).

[51] Guyon, O., Mazin, B., Fitzgerald, M., Mawet, D., Marois, C., Skemer, A., Lozi, J., and Males, J., "Wavefront control architecture and expected performance for the TMT Planetary Systems Imager," in [Adaptive Optics Systems VI], Society of Photo-Optical Instrumentation Engineers (SPIE) Conference Series 10703, 107030Z (July 2018). 